\title{
THE LENGTHENING PENDULUM
}

\author{
A. WERNER and C. J. ELIEZER
}

(Received 31 May 1967; revised 28 November 1967)

\section{Introduction}

Some recent papers have revived interest in some questions concerning the motion of a simple pendulum which is oscillating with small angular amplitude under gravity, when the length of the pendulum changes with time in some prescribed manner.

Littlewood, in a series of papers $([\mathbf{1}]-[5])$ has considered the adiabatic invariants which arise in a variety of problems where some quantity in each problem undergoes a slow change. In [1] he considers the pendulum of varying length and refers to the first Solvay Conference in 1911, when the problem of the shortening pendulum was raised by Lorentz. It was conjectured that if the equation of motion is taken as $\ddot{x}+\omega^{2} x=0$, where $\omega$ changes slowly with time, and the energy at time $t$ is taken as $E(t)=\frac{1}{2}\left(\dot{x}^{2}+\omega^{2} x^{2}\right)$, then $E / \omega$ is approximately constant. No rigorous proof had been forthcoming for this result or for other conjectures made in relation to this problem. that if

A rigorous treatment has now been provided by Littlewood. He showed

$$
H(t)=\frac{2 E}{\omega}, \quad \dot{x}=y, \quad\left|\omega^{(n)}\right|<\text { (constant) } \times \varepsilon^{n},
$$

where $\omega^{(n)}$ denotes the $n$-th derivative, then:

(i)

(1) $H(t)=H(\infty)-\frac{\dot{\omega}}{\omega^{2}} x y+\frac{1}{2}\left(\frac{\ddot{\omega}}{\omega^{4}}-\frac{2 \dot{\omega}^{2}}{\omega^{5}}\right)\left(y^{2}-\omega^{2} x^{2}\right)-\frac{1}{8} \frac{\dot{\omega}^{2}}{\omega^{4}} H(\infty)+O\left(\varepsilon^{2}\right)$.

(ii) The average of $H$ over $\left(t-\frac{1}{2} p, t+\frac{1}{2} p\right)$, where $p=2 \pi / \omega$ is the local period, is

$$
\mathscr{A}_{p}\{H(t)\}=\frac{1}{p} \int_{t-\frac{1}{2} p}^{t+\frac{1}{2} p} H(\tau) d \tau=H(\infty)+O\left(\varepsilon^{2}\right) .
$$

(iii) If $x=(H / \omega)^{\frac{1}{2}} \cos \alpha, y=-(H / \omega)^{\frac{1}{2}} \sin \alpha$ at time $t$, then the average over phase $\alpha$ is 
$\mathscr{A}_{\alpha}\{H(t)\}=\frac{1}{2 \pi} \int_{-\pi}^{\pi} H(t) d \alpha=-\frac{1}{8} \frac{\dot{\omega}^{2}}{\omega^{4}} H(\infty)+O\left(\varepsilon^{3}\right) \quad\left(-\frac{1}{2} p<t<\frac{1}{2} p\right)$. (iv)

$$
H(\infty)-H(-\infty)=O\left(\varepsilon^{n}\right) \text { for every } n .
$$

In physical terms, these relations include the conclusions: $H(t)-H(\infty)$ is of order $\varepsilon$; averaging over period improves this to order $\varepsilon^{2}$, with similar result for averaging over initial phase $\alpha$.

A case of the varying pendulum where the linearized equation of motion may be solved exactly in terms of known functions has been discussed recently by Brearley [6]. (See also Relton [7].) This occurs when $\dot{r}$ is constant, that is, when the length $r$ of the pendulum changes uniformly. Let $\dot{r}=V$. When the angular displacement $\theta$ from the vertical is expressed in terms of $r$, the solution obtained is

$$
\theta(r)=\frac{1}{\sqrt{ } r}\left\{C J_{1}(\lambda \sqrt{ } r)+D Y_{1}(\lambda \sqrt{ } r)\right\}
$$

where $\lambda=2 \sqrt{ } g / V, C$ and $D$ are arbitrary constants, and $J_{1}, Y_{1}$ are Bessel functions of order one of the first and second kind.

The physical implications of this solution are however worth examining. In particular, the variation of energy in the different stages of the motion needs to be studied.

In the present paper, the authors examine some of the physical consequences of the Brearley solution. The authors also report the existence of a family of cases where the equation of motion may be solved in terms of Bessel functions. The existence of this family of solutions does not appear to have been noticed before.

\section{The uniformly lengthening pendulum}

From the equations of motion

$$
\ddot{r}-r \dot{\theta}^{2}=-T+g \cos \theta, \quad r \ddot{\theta}+2 \dot{r} \dot{\theta}=-g \sin \theta
$$

one deduces, after substituting $r=l+V t$, where $l$ is the length at $t=0$, and approximating for small oscillations,

$$
r \frac{d^{2} \theta}{d r^{2}}+2 \frac{d \theta}{d r}+\frac{g \theta}{V^{2}}=0 .
$$

Writing $x= \pm \lambda \sqrt{ } r$ according as $V \gtrless 0$ (so that $x$ is always positive) and $y=x \theta$, one obtains

$$
x^{2} y^{\prime \prime}+x y^{\prime}+\left(x^{2}-1\right) y=0,
$$


which has solutions $J_{1}(x)$ and $Y_{1}(x)$. Thus, equation (7) has the solution (5).

The general features of the motion may be studied by taking a simpler form of the solution which is obtained when the initial conditions are such that at $t=0, \theta=0$ and $x= \pm 2 / V \sqrt{ } g l=x_{1}$ where $x_{1}$ is a positive zero of $J_{1}(x)$. For a given $V$, this may be realised by choosing appropriate value for $l$. The arbitrary constant $D$ then vanishes, and we have the solution

$$
\theta=\frac{1}{\sqrt{ } r} C J_{1}(\lambda \sqrt{ } r)
$$

If $x_{1}, x_{2}, x_{3}, \cdots$ are successive zeros of $J_{1}(x)$, and $X_{1}, X_{2}, X_{3}, \cdots$ successive zeros of $J_{2}(x)$, then at the vertical positions (given by $\theta=0$ ) the lengths of the pendulum are $r_{k}=x_{k}^{2} / \lambda^{2}(k=1,2, \cdots)$, and at the extreme positions (given by $\dot{\theta}=0$ ) the lengths are $R_{k}=X_{k}^{2} / \lambda^{2}(k=1,2, \cdots)$.

For the energy, we take

$$
E=\frac{1}{2}\left(\dot{r}^{2}+r^{2} \dot{\theta}^{2}\right)-g r\left(1-\frac{\theta^{2}}{2}\right)
$$

and derive, for the case of the lengthening pendulum with $V>0$, the following:

(i)

$$
T=\left[1+\frac{C^{2}}{x^{2}}\left(J_{0}^{2}-4 \frac{J_{0} J_{1}}{x}+4 \frac{J_{1}^{2}}{x^{2}}\right)\right] g,
$$

which tends to $g$ as $t \rightarrow \infty$.

(ii) $\frac{d E}{d t}=-T V<0$.

Hence the energy steadily decreases with time, and tends to $-\infty$ as $t \rightarrow \infty$.

(iii) The expression for $E$ takes the simple form

$$
E=\frac{1}{2} V^{2}-g r+\frac{V^{2} C^{2}}{8}\left(J_{1}^{2}+J_{2}^{2}\right)
$$

(iv) The extreme positions $\theta=\theta_{k}(k=1,2, \cdots)$ are such that $\left|\theta_{k}\right|<\left|\theta_{k-1}\right|$. Ultimately, $\theta_{k} \rightarrow 0$ as $k \rightarrow \infty$. Thus the angular amplitude decreases and tends to zero as $t \rightarrow \infty$. (However $r \theta_{k} \rightarrow \infty$ ).

(v) Meaning by a cycle the motion between two successive vertical positions with the pendulum moving in the same sense, the $k$-th cycle occurs between the values $x_{2 k-1}$ and $x_{2 k+1}$ for $r$. Denoting by $\tau_{k}$ the time of the $k$-th cycle,

$$
\tau_{k}=\frac{1}{V}\left(r_{2 k+1}-r_{2 k-1}\right)=\frac{V}{4 g}\left(x_{2 k+1}^{2}-x_{2 k-1}^{2}\right) .
$$


For large $k$, asymptotically

$$
\tau_{k} \sim \frac{V \pi}{g}\left(x_{2 k-1}+\pi\right), \quad \tau_{k+1}-\tau_{k} \sim \frac{2 \pi^{2} V}{g} .
$$

Hence the time for a cycle increases eventually by a constant quantity for each cycle.

(vi) The average energy for the $k$-th cycle is

$$
\begin{aligned}
& \bar{E}_{k}=\frac{1}{t_{2 k+1}-t_{2 k-1}} \int_{t_{2 k-1}}^{t_{2 k+1}} E d t \\
& =\frac{1}{2} V^{2}\left[1-\frac{1}{4}\left(x_{2 k+1}^{2}-x_{2 k-1}^{2}\right)+\frac{C^{2}}{2} \frac{\left\{x_{2 k+1}^{2} J_{0}^{2}\left(x_{2 k+1}\right)-x_{2 k-1}^{2} J_{0}^{2}\left(x_{2 k-1}\right)\right\}}{x_{2 k+1}^{2}-x_{2 k-1}^{2}}\right],
\end{aligned}
$$

which yields

$$
\bar{E}_{k} \sim \frac{1}{2} V^{2}\left[1-\pi\left(x_{2 k-1}+\pi\right)+\frac{C^{2} \cos ^{2}\left(x_{2 k-1}-(\pi / 4)\right)}{2 \pi\left(x_{2 k-1}+\pi\right)}\right],
$$

and

$$
\bar{E}_{k+1}-\bar{E}_{k} \sim-V^{2}\left[\pi+\frac{1}{2} C^{2} \cos ^{2}\left(x_{2 k-1}-\frac{\pi}{4}\right)\right] \sim-A,
$$

where $A$ is a positive finite constant. Thus for large $t$, the average energy decreases by a constant quantity for each cycle.

It would be instructive to compare the above results with those of Littlewood. Littlewood deals with problems of small change, and therefore comparison may be made only when $V$ is small, of order $\varepsilon$.

On substituting $x=r \theta$ in the equations of motion (7), one obtains

$$
\ddot{x}+\left(\frac{g}{r}-\frac{\ddot{r}}{r}\right) x=0
$$

so that in Littlewood's notation, $\omega^{2}=(g-\ddot{r}) / r=g / r$ for the uniformly changing pendulum. Further

$$
\begin{aligned}
H(t) & =\frac{C^{2}}{\lambda^{2}}\left(J_{0}^{2}+J_{1}^{2}\right) \sqrt{ } g r \\
H(\infty) & =C^{2} V / \pi \lambda^{2} .
\end{aligned}
$$

The energy expression (10) which we have used is however different from that used by Littlewood, which is $\frac{1}{2}\left(\dot{x}^{2}+\omega^{2} x^{2}\right)$. It appears to us that (10) is closer to the physical situation, while the latter relates to the energy of the equivalent constant length pendulum at each instant of time. The comparison is therefore not pursued further. 
In the case of the shortening pendulum, it has been pointed out by Brearley that owing to $Y_{1}(r)$ being unbounded as $r$ tends to zero, the approximation for small oscillation does not remain valid in the general case. The equations of motion here lead to a non-linear differential equation of the form

$$
\frac{\dot{d^{2} \theta}}{d u^{2}}=-\lambda^{2} \frac{\sin \theta}{4 u^{3}},
$$

where $u=1 / r$. The solution of such an equation as $u \rightarrow \infty$ should be of interest, and we hope to deal with it in a later paper.

\section{A family of soluble cases}

If $r=f(t)$, the equation corresponding to (7) is

$$
\theta^{\prime \prime}+\frac{f \ddot{f}+2 f^{2}}{f f^{2}} \theta^{\prime}+\frac{g}{f f^{2}} \theta=0 .
$$

This may be identified with the standard equation (See [8])

$$
\theta^{\prime \prime}+\frac{1-2 \alpha}{r} \theta^{\prime}+\left\{\left(\beta \gamma r^{\gamma-1}\right)^{2}+\frac{\alpha^{2}-p^{2} \gamma^{2}}{r^{2}}\right\} \theta=0
$$

which has the solution

$$
\theta=r^{a} Z_{p}\left(\beta r^{\gamma}\right),
$$

where $Z_{p}=C J_{p}+D Y_{p}$ and $p, \alpha, \beta, \gamma$ are constants.

We consider the following cases:

(a) $\gamma=1$. Identification then leads to either $f=$ constant or $f f^{2}=$ constant. From the latter we obtain $\alpha=-\frac{1}{4}, \beta=\sqrt{g / a}, p= \pm \frac{1}{4}$,

$$
f=\left\{\frac{3}{2}(\sqrt{a} t+b)\right\}^{\frac{2}{3}}, \quad \theta=\frac{1}{r t} Z_{ \pm \frac{1}{4}}(\sqrt{g / a} r) .
$$

(b) $\gamma \neq 1$ and $\alpha^{2} \neq p^{2} \gamma^{2}$. Then $f$ is constant.

(c) $\gamma \neq 1$ and $\alpha^{2}=p^{2} \gamma^{2}$. Then $\gamma=\frac{1}{2}(3+4 \alpha), p= \pm 2 \alpha /(3+4 \alpha)$ and $\alpha, \beta$ are arbitrary but such that

$$
f=\left[-2 \alpha\left\{\frac{2 \sqrt{ } g}{\beta(3+4 \alpha)} t+b\right\}\right]^{-\frac{1}{2} \alpha}
$$

is always real. Then

$$
\theta=r^{\alpha} Z_{2 \alpha /(1+3 \alpha)}\left\{\beta r^{(3+4 \alpha) / 2}\right\} .
$$

If $\alpha=1 / 2 n$ where $n$ is an integer, then $r$ is always real. Thus there exists a two-parameter family of solutions. 
When $\alpha=-\frac{1}{2}, \beta=2 \sqrt{g} / V=\lambda, \gamma=\frac{1}{2}, p= \pm 1$, we have $f=V t+b$, $\theta=(1 / \sqrt{ } r) Z_{1}(\lambda \sqrt{ } r)$ which gives the special case of the uniformly changing pendulum.

One of the authors (C. J. Eliezer) wishes to thank Professors G. B. Preston and R. Van der Borght and staff of the Mathematics Department of Monash University for hospitality.

\section{References}

[1] J. E. Littlewood, 'Lorentz's pendulum problem'. Annals of Physics 21(1963), 233-242.

[2] J. E. Littlewood, 'Abiadatic invariance II. Elliptic motion about a slowly varying centre of force'. Ibid. 26 (1964), $131-156$.

[3] J. E. Littlewood, 'Adiabatic invariance III. The equation $\ddot{x}=-V_{x}(x, \omega)$ '. Ibid. 29 (1964), 1-12.

[4] J. E. Littlewood, 'Adiabatic invariance IV. Note on a new method for Lorentz's pendulum problem'. Ibid. 29 (1964), 13-18.

[5] J. E. Littlewood, 'Adiabatic invariance V. Multiple periods'. Ibid. 30 (1964), 138-153.

[6] M. N. Brearley: 'The simple pendulum with uniformly changing string length'. Proc. Edin. Math. Soc. 15 (Ser. 2), Pt. 1 (1966), 61-66.

[7] F. E. Relton, Applied Bessel Functions. (Blackie, 1946), 56, 80.

[8] E. Jahnke \& F. Emde, Tables of Functions. (Dover, 1945), 146.

Melbourne, Australia

Monash University

Melbourne, Australia 